Original Research Article

\title{
Effectiveness of chitosan mouthwash on plaque formation and levels of salivary Streptococcus and Lactobacillus count: an in vivo study
}

\author{
Aparna Indu S. R. ${ }^{1}$, Sharan S. Sargod ${ }^{1}$, Sham S. Bhat ${ }^{1}$, \\ Shamjith Manikkoth $^{2}$, N. Ramakrishnan ${ }^{3}$
}

${ }^{1}$ Department of Paedodontics and Preventive Dentistry,

${ }^{2}$ Department of Pharmacology

Yenepoya Dental College

Yenepoya (deemed to be

University), Deralakatte,

Mangalore, Karnataka, India

${ }^{3}$ Department of Animal

Husbandry,

Thiruvananthapuram

Kerala, India

Received: 20 July 2019

Revised: 13 October 2019

Accepted: 14 October 2019

*Correspondence to:

Dr. Aparna Indu S. R.,

Email: aparnaindu077@

gmail.com

Copyright: (C) the author(s), publisher and licensee Medip Academy. This is an openaccess article distributed under the terms of the Creative Commons Attribution NonCommercial License, which permits unrestricted noncommercial use, distribution, and reproduction in any medium, provided the original work is properly cited.

\begin{abstract}
Background: The objectives of the present study were to clinically evaluate the effectiveness of chitosan mouthwash on Plaque formation and to evaluate the antimicrobial activity against salivary Streptococcus mutans (SM) and Lactobacillus (LB).

Methods: A total of 20 participants with decay-missing-filled index $\geq 4$ and simplified oral hygiene index score $>1.3$ were recruited for the study after taking informed consent. All the participants were provided a bottle of chitosan mouthwash in addition to their usual oral hygiene procedure. Baseline salivary SM and LB levels were determined, using chair-side CRT® bacteria test prior giving chitosan mouthwash and after 14 days, during which children are advised to use $10 \mathrm{ml}$ of mouthwash for 60 seconds two times daily. Plaque index score recorded using Silness and Loe plaque index. Paired t-test (or corresponding non-parametric) and percentage comparison method using cross tables were used for statistical analysis.

Results: The baseline plaque index score of 0.758 and after 14 days of mouthwash use, it reduced to 0.434 . The mean difference in the plaque index showed significant reduction in the plaque score (i.e., with the difference of 0.324). The results showed the antiplaque effects with a short duration of 14 days use of chitosan $(\mathrm{CH})$ mouthwash. The antibacterial activity demonstrated a range of inhibitory effect on salivary SM and LB.

Conclusions: $\mathrm{CH}$ showed an evident strong effect against salivary SM and LB levels and controlling the plaque biofilm formation. So water soluble $\mathrm{CH}$ can be used in new formulations for oral applications not only as antimicrobial agent but also for plaque biofilm control.
\end{abstract}

Keywords: Chitosan, SM, LB, Mouthwash, Plaque, In vivo

\section{INTRODUCTION}

Streptococcus mutans (SM), Lactobacillus (LB) are the micro-organisms responsible for initiation of dental caries. Dental caries is associated by plaque as it develops and matures. Hence plaque control is of paramount importance in control both dental caries and periodontal problems. Plaque control includes mechanical and chemical. Mechanical plaque control is by using proper tooth brushing techniques. Chemically among the various topical antimicrobial agents mouthwash has been particularly well accepted due their ease of use.

Mouthwashes (mouth rinses) are solutions or liquids used to rinse the mouth for a number of purposes to remove or destroy bacteria, to act as an astringent, to refresh and to 
have a therapeutic effect by relieving infection or preventing dental caries. Chlorhexidine $(\mathrm{CHX})$ is regarded as gold standard in plaque control, but side effects such as discoloration of the teeth and the tongue, altered taste sensation, interfering against oral micro-flora and increased calculus formation after its use for long periods is noted. ${ }^{2}$ Because of these background, there is a need for a new product which has same or/ more efficient than conventional mouthwash and decreases the adverse effects of conventional mouthwash.

Chitosan $(\mathrm{CH})$ is a natural co-polymer of chitin with specific characteristics, including biodegradability, bioadhesive, biodegradable, non-toxicity antifungal and antimicrobial activity, composed by units of 2-amino-2desoxi-D-glycopyranose and of 2-acetamide-2-desoxi-Dglycopyranose interconnected by glycosidic bonds $\beta-1,4$ in variable proportions. $\mathrm{CH}$ is prepared by the de-Nacetylation of chitin. It acts with negatively charged bacterial cell membrane and cause leakage of proteinaceous and other intra cellular constituents thereby alters cell permeability. As a bioadhesive polymer, $\mathrm{CH}$ provides an extended withholding time on the oral mucosa.

The great advantages of $\mathrm{CH}$ is contamination of proteins are absent but there are chances proteins could cause allergic reactions in individuals with shellfish allergies. There are no reported cases of other side effects till now.

This in vivo study aimed to investigate the effectiveness of $\mathrm{CH}$ mouthwash on plaque formation, and antimicrobial activity against salivary SM and LB count.

\section{METHODS}

A pilot study was carried out at the Department of Pedodontics and Preventive dentistry during the period of December 2016 to May 2018 in Yenepoya Dental College (Yenepoya Deemed to be University) Managalore after obtaining ethical clearance from institution ethical committee, children aged 8-12 years were selected from a residential school who are residing in the same institution, thereby eliminating the bias occurring due to different eating patterns. A written informed consent was obtained from all parents before enrolling into the study. An initial screening was done for all the children. decay-missing-filled index (DMFT) and simplified oral hygiene index score (OHI-S) index were recorded. Children with DMFT $\geq 4$ and OHI-S score $>1.3$ recruited for the study.

Each volunteer had to fulfill the following inclusion criteria as be in good general health; age group of 8-12 years with no history of steroid or antimicrobial therapy in last 3 months, no regular medication containing antiinflammatory compounds; not be using tobacco products; have no regular use of oral antiseptics; have a minimum of 12 gradable teeth and fair oral hygiene and have no fixed or removable prostheses, or orthodontic appliances were recruited for the study. Those reported to be allergic to $\mathrm{CH}$ derivates were not allowed to participate and developing severe gingival inflammation during the study were excluded. The study protocol is summarized in Figure 1.

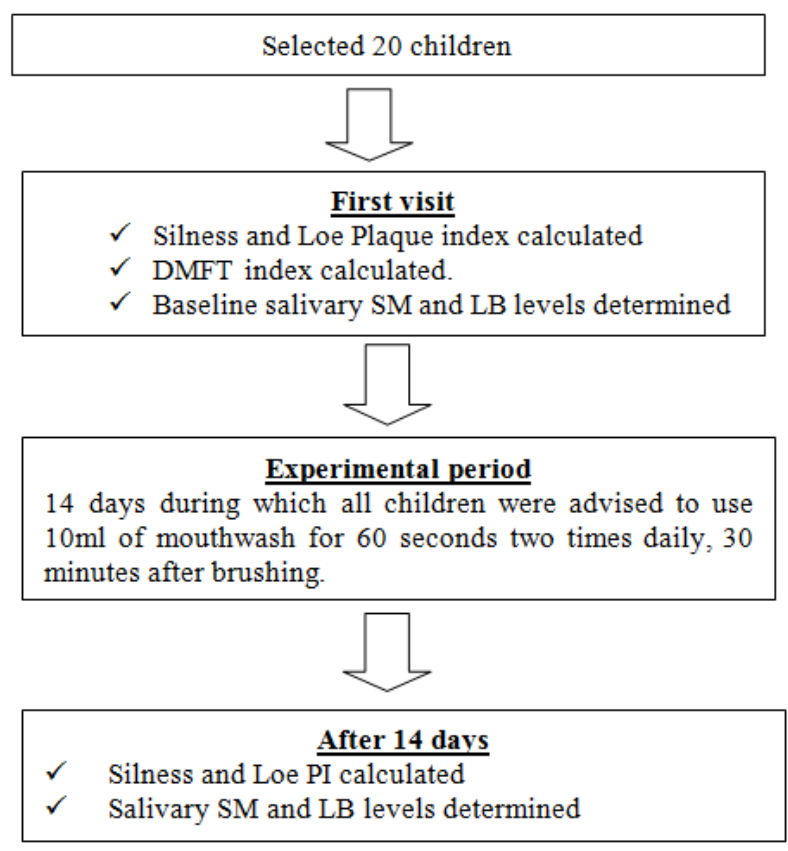

Figure 1: Study protocol.

Normality assumption checked. Paired t-test (or corresponding non-parametric) was used for plaque biofilm formation and for SM, LB levels. Percentage comparison method using cross tables were used for the comparison of change from baseline to 14 days intervals in various parameters (intra group) (Table 3, Figures 4 and 5).

\section{Preparation of $\mathrm{CH}$ mouthwash}

Water soluble $\mathrm{CH}$ powder (Blueline food India Pvt ltd) procured commercially. $0.5 \%$ water soluble $\mathrm{CH}$ mouthwash was prepared freshly in Department of Pharmacology (Table 1) (Figure 2). ${ }^{1-3}$

\section{Assessment of plaque accumulation}

Plaque scoring by the OHI-S is calculated by adding debris index simplified (DI-S) and calculus index simplified (CI-S). All examinations were performed by a single experienced dental examiner.

\section{Estimation of the level of mutans streptococci and LB in saliva}

The levels of SM and LB were evaluated using commercial caries risk test (CRT) kit. A drop of saliva was pipetted on each surface, one for SM and other for LB. The agar plates were incubated using an incubator 
for 48 hours at $37^{\circ} \mathrm{C}$ to allow the growth of the organisms following manufacturer's instructions. Growth of the bacteria was evaluated under good lighting conditions. Bacterial growth was then scored by comparing with standards expressed in colony forming units (CFUs) provided by the manufacturer (Table 2, Figure 3 ).

\section{RESULTS}

Present study started (Table 3) with an average baseline Silness and Loe plague index (PI) score of 0.7575 and after 14 days of mouthwash use it reduced to 0.4343 . The mean difference in the plaque index showed significant reduction in the plaque score (i.e., with the difference of 0.3235 ) with a standard deviation of 0.984174 . The results showed the antiplaque effects with a short duration of 14 days of use of $\mathrm{CH}$ based mouthwash. However, when the Sillness and Loe PI scores were compared, one of the best antiplaque effects was obtained within 14 days use of water soluble $\mathrm{CH}$ mouthwash.

Table 1: Ingredients of CH mouthwash.

\begin{tabular}{|ll|}
\hline Ingredients & Composition \\
\hline Chitosan & $1 \mathrm{~g}$ \\
\hline Substance S* & $25 \mathrm{~g}$ \\
\hline Vanilla flavor & 20 drops \\
\hline Water & $280 \mathrm{ml}$ \\
\hline
\end{tabular}

Substance $\mathrm{S}^{*}$ is an inert material without any biological activity.

Table 2: CFUs/ml SM and LB.

\begin{tabular}{|ll|}
\hline Low & Less than $105 \mathrm{CFUs} / \mathrm{ml}$ \\
\hline High & Greater than $10^{5} \mathrm{CFUs} / \mathrm{ml}$ \\
\hline
\end{tabular}

Table 3: Baseline and after 14 days Silness and Loe PI score's mean and standard deviation.

\begin{tabular}{|llll|}
\hline & Before & After & Difference \\
\hline Mean & 0.7575 & 0.4343 & 0.3235 \\
\hline S.D. & 0.503854 & 0.46647 & 0.984174 \\
\hline
\end{tabular}

Percentage comparison method using cross tables were used for the comparison of antibacterial activity of SM and LB from baseline to 14 days intervals of water soluble $\mathrm{CH}$ mouthwash use (intra group). Evaluated using simple chair-side CRT bacteria kit and the findings demonstrated a range of inhibitory effect on salivary SM of $50 \%$ and LB of $55 \%$ (Figures 4 and 5).

Statistical analysis of this study indicated that the water soluble $\mathrm{CH}$ mouthwash demonstrated a range of inhibitory effects on salivary SM and LB. Also it showed reduction in plaque biofilm formation. These conclusions were supported by both the clinical parameters and microbiologic outcomes.

From Table 3,
Mean under head difference is not the difference of means but the mean of differences obtained from the observations. Same as, it is not the difference of standard deviation (SD) but it is the SD of the differences obtained from the observations.

Calculated $\mathrm{t}=\overline{\mathrm{d}} \times \sqrt{\mathrm{n}} / S=0.3235 \times \sqrt{ } 20 / 0.984174=1.47$

Calculated $\mathrm{t}$ value $=1.47$

Where $\overline{\mathrm{d}}$ is the mean of differences, $\mathrm{s}$ the SD and $\mathrm{n}$ the sample size. Which follows students $\mathrm{t}$ distribution with (n-1) degrees of freedom. ${ }^{4}$
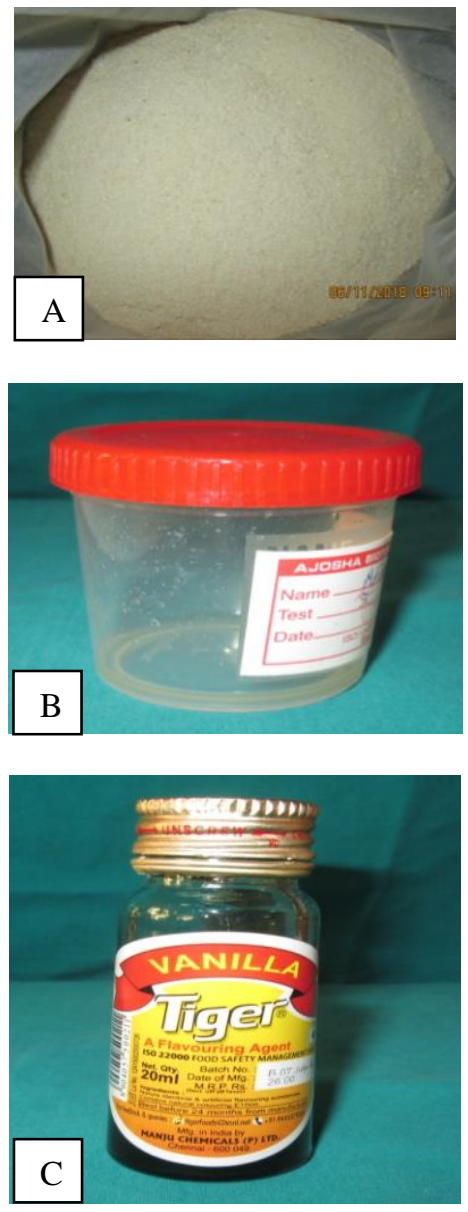

Figure 2 (A): Water soluble $0.5 \% \mathrm{CH}$ powder; (B): Collected saliva and (C): Vanilla flavour.

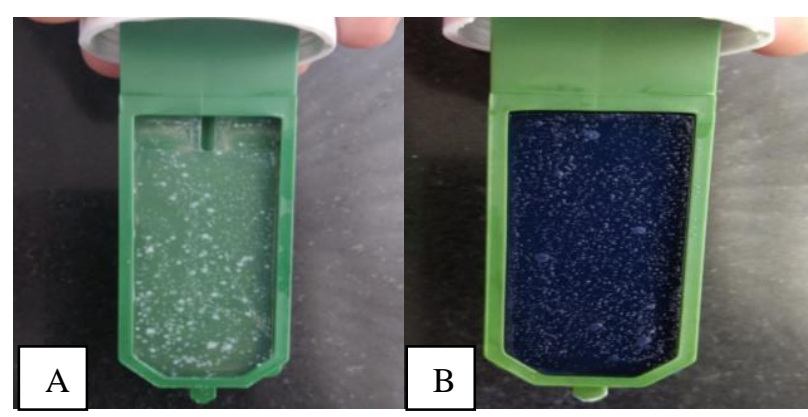

Figure 3 (A): Growth of LB and (B): Growth of SM. 


\section{Statistical inference}

Since calculated t value is less than table value, we accept the null hypothesis and conclude that $\mathrm{CH}$ mouthwash reduces plaque bio-film formation.

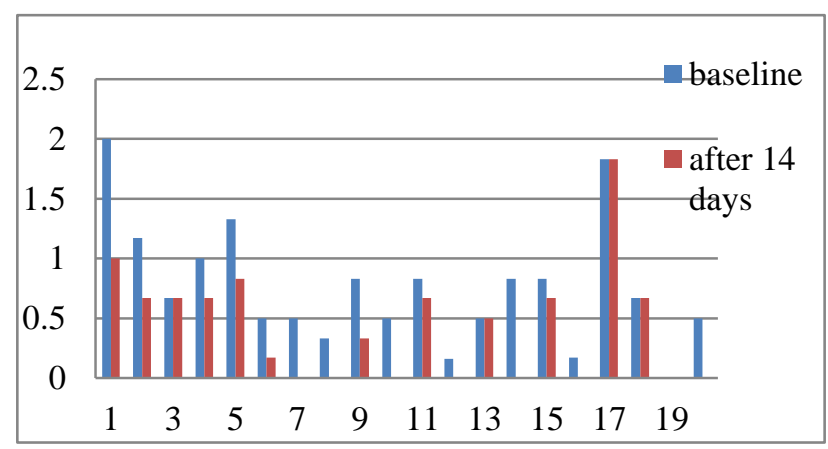

Figure 4: PI baseline and after 14 days of rinsing.
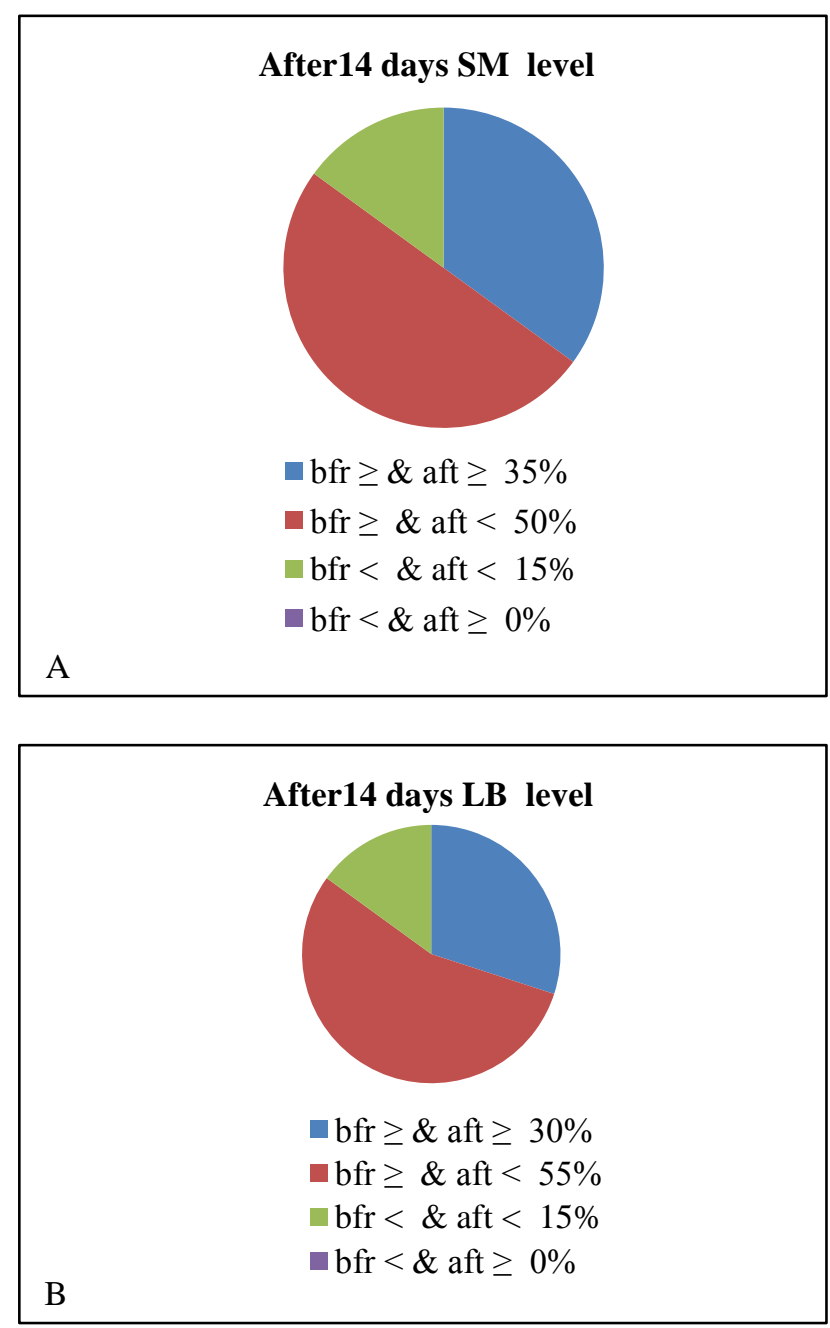

Figure 5: (A) Levels of SM and (B) LB after 14 days.

\section{DISCUSSION}

$\mathrm{CH}$ is a biocompatible, biodegradable to normal body constituents, safe and non-toxic natural co-polymer found in the cell wall of fungi, crab and prawn shells. A wide variety of medical application of $\mathrm{CH}$ and chitin derivatives has been reported over the last three decades. Apart from their application in medical field $\mathrm{CH}$ fibers have potential applications in waste water treatment. For different applications, different properties of $\mathrm{CH}$ are used. $\mathrm{CH}$ has got an extended withholding time on the oral mucosa with antibacterial and anti-plaque activity and absence of any reported side effects in the present literature; we used $\mathrm{CH}$ for the preparation of mouthwash formulation.

The aim of the study was to clinically evaluate the effectiveness of $\mathrm{CH}$ mouthwash on plaque formation, and its antimicrobial activity against Salivary SM and LB. SM and LB are microorganisms that are primarily associated with dental caries.

CHX, a cationic bisbiguanide has very wide antimicrobial spectrum and is a broadly used over the counter mouth rinse has been accepted as gold standard. The major advantage of $\mathrm{CHX}$ is its substantivity, while binding into the soft and hard tissues enabling it to act over a long period after application. However, $\mathrm{CHX}$ has several side effects, such as staining and alteration of taste, which limit its extended use. ${ }^{5-7}$

Kowitz et al had reported the irritating effect of $\mathrm{CHX}$ mouthwashes to the oral mucosa by demonstrating the occurrence of epithelial peeling, gingivitis, petechiae, mucosal ulceration associated with inflammation. ${ }^{8}$ In a review article Gagari et al explained the local and systemic side effects associated with continuous use of mouthwash. ${ }^{9}$ Diffuse whiteness with fissuring and desquamation of mucosa, type I and IV hypersensitivity reactions are common.

$\mathrm{CH}$ is a natural polymer with different specific characteristics, including biodegradability, bioadhesive, non-toxicity, antifungal and antimicrobial activity. ${ }^{10}$ Several researchers demonstrated that this polysaccharide has antimicrobial action in a great variety of microorganisms, included gram-positive bacteria and various species of yeast. ${ }^{11}$ It also provides an extended withholding time on the oral mucosa.

Stamford et al explained about the potential application of $\mathrm{CH}$ as an anticariogenic agent in different formulations such as toothpaste (Chitodent $\left.{ }^{\circledR}\right)$, mouthwash solution and chewing gum. ${ }^{11}$ Chitodent ${ }^{\circledR}$ is a homeopathically compliant formulation toothpaste, which was developed in Emsland, Germany. $\mathrm{CH}$ is the active ingredient and is free of salicylate and fluoride. It is now the only natural biological toiletry product available in the global market with a BDIH certificate. Chen et al found that the antibacterial effect of $\mathrm{CH}$ was similar to the commercially available mouthwashes through an in-vivo and in vitro studies using different $\mathrm{pH}(\mathrm{pH}$ 5-8) and temperatures $\left(25-37^{\circ} \mathrm{C}\right) .^{3}$ 
The effect of mouthwash used to be confounded by different variables like Hawthorne effect, improved oral hygiene, pre-prophylaxis, interactions between mouth rinse and ingredients of other oral hygiene aids. This study avoided maximum of such factors influences by using normal home care hygiene habits, thereby the possible better results of $\mathrm{CH}$ mouthwash. ${ }^{2}$

Most of the commercially available mouthwashes use alcohol as a vehicle. Weaver et al first reported about the chances of oral cancer due to continuous use of alcohol containing mouthwashes. ${ }^{11}$ Wynder et al, Blot et al, Mashberg et al, Kabat et al and Winn et al also supported these findings. ${ }^{13-17}$ Lemos et al in his review article mentioned about the burning or sore sensations, xerostomia and chances of accidental ingestion of mouthwashes in children. ${ }^{17,18}$ In this study, water was used as a vehicle in the mouthwash formulation to avoid side effects caused due to alcohol. ${ }^{3}$

$0.5 \% \mathrm{CH}$ formulation was used in this study because the formulations prepared by Sano et al, Archana et al, Costa et al, Rahmani et al, Shah et al showed maximum anticariogenic activity which was similar to this study. However the methodology and the parameters considered were different. ${ }^{1,2,20-22}$

Saliva was used as a parameter for assessment of SM and LB levels, because of ease of collection, it contains almost all the oral micro flora and it provides information on the component cause for the caries process. ${ }^{23}$

In this study we used a rinsing period of 14 days twice daily using $10 \mathrm{ml}$ of $\mathrm{CH}$ mouthwash for 60 seconds, which is similar to Uraz et al and Sano et al. ${ }^{2,24}$ The samples were collected at the baseline and at the end of 14 days after the use of $\mathrm{CH}$ mouthwash for assessing SM and LB levels using CRT kit. ${ }^{2}$

In the modern era of early detection and non-invasive caries management, there is a need for rapid and accurate biomarkers for caries activity and future caries risk. Twetman compared CRT® Bacteria (Ivoclar Vivadent) with Dentocult. ${ }^{25}$ CRT® Bacteria (Ivoclar Vivadent) is a simple chair side test to assess salivary SM and LB levels. It showed high sensitivity that is it can detect even lower count of SM. and also gives more specific results. The main advantage of using CRT kit is that, there is no need of any special apparatus or technique. Also it has the added advantage of being capable of evaluating two bacteria together within a short period of time. ${ }^{26}$

It was observed that the average baseline Sillness and Loe PI score was 0.758 and after 14 days of mouthwash use it was reduced to 0.434 (Table 3 ). The in vivo clinical trial showed the antiplaque effect with the short duration of 14 days of use of $\mathrm{CH}$ mouthwash by stimulation of saliva production thereby inhibiting the bacterial plaque formation. $\mathrm{CH}$ interactions with bacteria are very complex, depending on the characteristics of both the $\mathrm{CH}$ structure and the micro-organism surface. Also suggest that the polyampholyte nature of $\mathrm{CH}$ could be the necessary requisite for the expression of the generalized anti-adsorption effect. When the concentration of $\mathrm{CH}$ increases the anti-adhesion effect also increases and 5 $\mathrm{mg} / \mathrm{ml}$ was found to be the most effective anti-adhesive concentration. CH's high positive charge being capable of interacting and interfering with the biofilm structure by inhibiting biofilms formed by two micro-organisms and was capable of acting on mature biofilm leading to significant reduction in biofilm survival. $\mathrm{CH}$ also reduces biofilm viable cell numbers of Candida albicans metabolic activity by $70 \%$ (in 30 minutes) and $85.5 \%$ (in 24 hours) and biofilm viability by more than $90 \%$ (in 24 hours). ${ }^{11,23,27,28}$

From the results of this study it is found that daily rinsing with water soluble $\mathrm{CH}$ mouthwash controls plaque biofilm formation and significantly reduces the level of salivary SM (50\%) and LB (55\%) (Figure 7) without interfering or affecting the normal oral micro flora. Antibacterial activity of $\mathrm{CH}$ arises from a combination of both bacteria cell binding and deoxyribonucleic acid binding mechanisms. Low molecular weight $\mathrm{CH}$ is very effective at low concentration in reducing SM adsorption to hydroxyapetite and enhancing detachment without impairing the adhesive property of other streptococci. In case of high molecular weight $\mathrm{CH}$, it forms films around the cell that inhibit absorption of nutrients. The extended withholding time of $\mathrm{CH}$ helps it to acts with negatively charged bacterial cell membrane and cause leakage of proteinaceous and other intra cellular constituents thereby alters cell permeability. Smaller the size of $\mathrm{CH}$, it exhibited higher affinity for bacterial cell.

During the complete course of rinsing regimen none of the participants reported any side effects or adverse reactions. And also in the present literature there is no reports regarding any side effects caused due to $\mathrm{CH}$ formulations until and unless the patient is allergic to sea food.

Within the limitation of the study, $\mathrm{CH}$ showed strong antibacterial effect against salivary SM and LB levels and also controlling the plaque biofilm formation. Hence, water soluble $\mathrm{CH}$ can be used in new formulations for oral applications not only as antimicrobial agent but also for biofilm control. Further long-term clinical studies are required to prove the absence of side effects.

\section{Limitations}

The oral hygiene aids used by the patients were not taken into consideration and the effect on the normal flora of the oral cavity on long-term use was not assessed.

\section{Recommendations}

Long-term clinical studies are required to prove the absence of any side effects and complications. Possible 
use along with other oral hygiene aids. Added advantages of $\mathrm{CHX}$ and $\mathrm{CH}$ combinations if any and studies with different concentrations and/duration of use in children and adults.

\section{CONCLUSION}

Within the limitation of the study, $\mathrm{CH}$ showed strong antibacterial effect against salivary SM and LB levels and also controlling the plaque biofilm formation. So water soluble $\mathrm{CH}$ can be used in new formulations for oral applications not only as antimicrobial agent but also for plaque biofilm control. This study also suggests that $\mathrm{CH}$ may be an alternative natural agent for managing biofilm formation and levels of different cariogenic bacterias in oral cavity and it can be used as an active ingredient in various mouth wash formulations.

\section{ACKNOWLEDGEMENTS}

I am thankful to Mr. Hashim A, Department of Forensic Medicine and Toxicology, Yenepoya Medical College, Mangalore, Karnataka for helping me in the preparation of this article.

\section{Funding: No funding sources}

Conflict of interest: None declared

Ethical approval: The study was approved by the Institutional Ethics Committee

\section{REFERENCES}

1. Archana V, Prabhuji ML, Karthikeyan BV, Selvan A. Control of Streptococcus sanguinis oral biofilm by novel chlorhexidine-chitosan mouthwash: an in vitro study. J Exp Integrative Med. 2013;3(2).

2. Sano H, Shibasaki Ki, Matsukubo T, Takaesu Y. Effect of chitosan rinsing on reduction of dental plaque formation. Bulletin Tokyo Dent Coll. 2003;44(1):9-16.

3. Chen CY, Chung YC. Antibacterial effect of watersoluble chitosan on representative dental pathogens Streptococcus mutans and Lactobacilli brevis. J Appl Oral Sci. 2012;20(6):620-7.

4. Gupta SC, Kapoor VK. Fundamentals of mathematical statistics. Delhi, India: Sulthan Chand and Sons Publications Pvt Ltd; 2002.

5. Nagappan N, John J. Antimicrobial efficacy of herbal and chlorhexidine mouth rinse: a systematic review. J Dent Med Sci. 2012;2(4):5-10.

6. Van Leeuwen MP, Slot DE, Van der Weijden GA. Essential oils compared to chlorhexidine with respect to plaque and parameters of gingival inflammation: a systematic review. J Periodontol. 2011;82(2):174-94.

7. Flotra L, Gjermo Pe, Rolla G, Waerhaug J. Side effects of chlorhexidine mouth washes. Europ J Oral Sci. 1971;79(2):119-25.

8. Kowitz GM, Lucatorto FM, Cherrick HM. Effects of mouthwashes on the oral soft tissues. J Oral Med. 1976;31(2):47-50.
9. Gagari E, Kabani S. Adverse effects of mouthwash use: a review. Oral Surg Oral Med Oral Pathol Oral Radiol Endodontics. 1995;80(4):432-9.

10. Aliasghari A, Khorasgani MR, Vaezifar S, Rahimi F, Younesi H, Khoroushi M. Evaluation of antibacterial efficiency of chitosan and chitosan nanoparticles on cariogenic streptococci: an in vitro study. Iran $\mathbf{J}$ Microbiol. 2016;8(2):93.

11. Stamford TC, Stamford-Arnaud TM, de Medeiros Cavalcante HM, Macedo RO, de Campos-Takaki GM. Microbiological chitosan: Potential application as anticariogenic agent. In Practical Applications Biomed Engineer; 2013.

12. Weaver A, Fleming SM, Smith DB. Mouthwash and oral cancer: carcinogen or coincidence?. J Oral Surg (Am Dent Assoc: 1965). 1979;37(4):250-3.

13. Wynder EL, Kabat GC. Oral cancer and mouthwash use: reply. J Natl Cancer Institute. 1983;71(6):1105.

14. Blot WJ, Winn DM, Fraumeni JF. Oral cancer and mouthwash. J Natl Cancer Institute. 1983;70(2):2513 .

15. Mashberg A, Barsa P, Grossman ML. A study of the relationship between mouthwash use and oral and pharyngeal cancer. J Am Dent Assoc (1939). 1985;110(5):731-4.

16. Kabat GC, Hebert JR, Wynder EL. Risk factors for oral cancer in women. Cancer Res. 1989;49(10):2803-6.

17. Winn DM, Blot WJ, McLaughlin JK, Austin DF, Greenberg RS, Preston-Martin S, et al. Mouthwash use and oral conditions in the risk of oral and pharyngeal cancer. Cancer Res. 1991;51(11):3044-7.

18. Lemos-Junior CA, Villoria GE. Reviewed evidence about the safety of the daily use of alcohol-based mouthrinses. Brazil Oral Res. 2008;22:24-31.

19. Massey CC, Shulman JD. Acute ethanol toxicity from ingesting mouthwash in children younger than age 6, 1989-2003. Pediatric Dentist. 2006;28(5):4059.

20. Costa EM, Silva S, Madureira AR, Cardelle-Cobas A, Tavaria FK, Pintado MM. A comprehensive study into the impact of a chitosan mouthwash upon oral microorganism's biofilm formation in vitro. Carbohydrate Polymers. 2014;101:1081-6.

21. Rahmani F, Moghadamnia AA, Kazemi S, Shirzad A, Motallebnejad M. Effect of $0.5 \%$ Chitosan mouthwash on recurrent aphthous stomatitis: a randomized double-blind crossover clinical trial. Electronic physician. 2018;10(6):6912.

22. Shah HP, Bhavsar NV, Chavda MG. Comparative evaluation of clinical efficacy of chlorhexidine, phenolic and chitosan mouth rinses on plaque and gingivitis: single-centre, double blind, randomized controlled clinical study. Austin J Dent. 2017; 4(1): 1064 .

23. De Carvalho MM, Stamford T, Pereira E, Dos Santos P, Sampaio F. Chitosan as an oral antimicrobial agent. Formatex. 2011;2012(1):13.

24. Uraz A, Boynuegri D, Ozcan G, Karaduman B, Uc D, Senel S, et al. Two percent chitosan mouthwash: 
A microbiological and clinical comparative study. J Dent Sci. 2012;7(4):342-9.

25. Twetman L, Twetman S. Comparison of two chairside tests for enumeration of Streptococci mutans in saliva. Oral Health Dent Manag. 2014;13(3):580-3.

26. Walsh LJ, Tsang AK. Chairside testing for cariogenic bacteria: current concepts and clinical strategies. Int Dentistr South Africa. 2008;10(2):50-65.

27. Dutta PK, Dutta J, Tripathi VS. Chitin and chitosan: chemistry, properties and applications. J Scientific Industrial Res. 2004;63:20-31.
28. Decker EM, Von Ohle C, Weiger R, Wiech I, Brecx M. A synergistic chlorhexidine/chitosan combination for improved antiplaque strategies. J Periodont Res. 2005;40(5):373-7.

Cite this article as: Indu ASR, Sargod SS, Bhat SS, Manikkoth S, Ramakrishnan N. Effectiveness of chitosan mouthwash on plaque formation and levels of salivary Streptococcus and Lactobacillus count: an in vivo study. Int J Basic Clin Pharmacol 2019;8:2416-22. 